\title{
Registros da fauna de Collembola (Arthropoda, Hexapoda) no Estado da Paraíba, Brasil
}

\author{
Bruno Cavalcante Bellini ${ }^{1} \&$ Douglas Zeppelini ${ }^{2,3}$
}

\begin{abstract}
${ }^{1}$ Departamento de Sistemática e Ecologia, Centro de Ciências Exatas e da Natureza, Universidade Federal da Paraíba - Campus I. 58059-900, João Pessoa-PB, Brazil. entobellini@yahoo.com.br

${ }^{2}$ Centro de Ciências Biológicas e Sociais Aplicadas, Universidade Estadual da Paraíba Campus V, João Pessoa-PB, Brasil.

${ }^{3}$ Associação Guajiru - Ciência - Educação - Meio Ambiente.
\end{abstract}

\begin{abstract}
Records of collembolan fauna (Arthropoda, Hexapoda) in Paraíba State, Brazil. In this paper the species found in Paraíba State, northeatern Brazil are listed. The specimens were collected in six municipalities: João Pessoa, Cabedelo, Mataraca, Bananeiras, Cacimba de Dentro and Araruna. The specimens were collected using Berlese-Tullgren funnels, pitfall traps and entomological aspirators. After that they were mounted in glass slides for identification under microscope. It was found 54 species of collembolans, distributed in 25 genera, of 13 families. Entomobryidae was the most diverse family, with 22 species and Seira was the prevailing genus, with 15 species. Most species were found in fragments of Mata Atlântica and Mata de Restinga. Twenty new species were found and will be describe later. The data indicate that Paraíba is possibly a hotspot of diversity for the genus Seira.
\end{abstract}

KEYWORDS. Brazil; Collembola; edaphic fauna; species checklist.

RESUMO. Registros da fauna de Collembola (Arthropoda, Hexapoda) no Estado da Paraíba, Brasil. No presente trabalho são listadas as espécies de Collembola encontradas no Estado da Paraíba, Região Nordeste do Brasil. Os espécimes foram coletados em seis municípios: João Pessoa, Cabedelo, Mataraca, Bananeiras, Cacimba de Dentro e Araruna. Os espécimens foram coletados através do processamento de material em funis de Berlese-Tullgren, armadilhas do tipo pitfall e aspiradores entomológicos. Em seguida foram montados entre lâminas e lamínulas de vidro para identificação sob microscópio. Foram identificadas 54 espécies de colêmbolos, distribuídas em 25 gêneros, em 13 famílias. Entomobryidae foi a mais diversa, com 22 espécies e Seira foi o gênero mais diverso, com 15 espécies. A maior parte das espécies foi encontrada em remanescentes de Mata Atlântica e Mata de Restinga. Foram encontradas 20 novas espécies de colêmbolos e serão descritas posteriormente. Os dados aqui apresentados indicam que a Paraíba é um hotspot de diversidade para o gênero Seira.

PALAVRAS-CHAVE. Brasil; Collembola; fauna edáfica; lista de espécies.

Colêmbolos são microartrópodes terrestres tradicionalmente considerados como hexápodes basais (Kristensen 1981). Entretanto, estudos de morfologia, ontogenia e aspectos biomoleculares indicam que Collembola está mais relacionado com "Crustacea" do que com Insecta (Bellinger et al. 1996-2008).

Apesar de habitarem diversos tipos de habitats, colêmbolos são animais intimamente relacionados ao solo. Esses animais estão entre os artrópodes terrestres mais abundantes, juntamente com os ácaros (Miranda-Rangel \& Palacios-Vargas 1992; Zeppelini \& Bellini 2004). Colêmbolos se alimentam principalmente de fungos, podendo também consumir bactérias, detritos vegetais e animais (Cassagne et al. 2003; Castaño-Meneses et al. 2004). Isso faz com que sejam extremamente importantes na ciclagem de nutrientes no solo. Populações de colêmbolos eventualmente atuam como controladores da biomassa de fungos presentes no solo, assim como podem servir de vetores de dispersão para os mesmos (Kooistra 1964; Cassagne et al. 2003). Seu tamanho diminuto faz com que se tornem presas para outros pequenos artrópodes. Assim, integram a base da cadeia trófica ao servir de alimento para vários animais, especialmente insetos e aracnídeos em início do desenvolvimento (Christiansen \& Bellinger 1980, 1998).

Algumas espécies de Collembola são indicadoras de qualidade de solo (Bellinger et al. 1996-2008). A presença (ou ausência) de algumas espécies pode estar relacionada com alterações no $\mathrm{pH}$, disponibilidade de determinados íons e água, presença de componentes de pesticidas ou mesmo cargas de metais pesados presentes em solos contaminados (Cassagne et al. 2003, 2004; Choi \& Moorhead 2006). As espécies endêmicas também podem ser indicativas de impactos ambientais (Frampton 2000).

Até o momento foram descritas mais de 7600 espécies de Collembola, sendo a maior parte registrada em regiões de clima temperado. Entretanto, estima-se que uma maior diversidade do grupo esteja na região Neotropical. Atualmente, para essa região, totaliza-se pouco mais de 1200 espécies registradas (Mari-Mutt \& Bellinger 1990, 1996; Mari-Mutt et al. 1998-2008; Bellinger et al. 1996-2008). O reduzido número de espécies reconhecidas para a região não condiz com sua grande diversidade de habitats. A baixa diversidade registrada para a região reflete um baixo esforço de coleta (Bellini \& Zeppelini 2005). No Brasil foram registradas 223 espécies, representando 
80 gêneros em 19 famílias (Zeppelini \& Bellini 2004; Fernandes \& Mendonça 2004; Abrantes \& Mendonça 2005, 2007; Bellini \& Zeppelini 2005; Zeppelini 2006; Mendonça \& Fernandes 2007). Esse número é certamente incapaz de refletir a composição faunística existente no país (Culik \& Zeppelini 2003).

\section{MATERIALE MÉTODOS}

Os espécimes foram coletados entre 2002 e 2008, no Estado da Paraíba, nos municípios de João Pessoa, Araruna, Cacimba de Dentro, Cabedelo, Mataraca e Bananeiras (Fig. 1). Em João Pessoa, as coletas foram realizadas na Mata do Buraquinho, no Campus I da Universidade Federal da Paraíba, na Costa do Sol e na Praia da Penha; em Araruna, no Parque Ecológico da Pedra da Boca; em Cacimba de Dentro, na fazenda Cachoeira da Capivara; em Cabedelo, na praia Mar do Macaco; em Mataraca, na empresa de mineração Lyondell Chemicals; e em Bananeiras, na periferia da cidade.

As coletas foram feitas por amostragem de folhiço e solo e posterior processamento desse material em funis de BerleseTullgren; através de armadilhas do tipo pit-fall de tamanhos variados (variando entre 50 e 2000ml) contendo álcool 70\%; e coletas diretas, com utilização de aspiradores entomológicos.

Os espécimes foram triados sob microscópio estereoscópico e armazenados em frascos do tipo Eppendorf, contendo álcool $70 \%$. Em seguida, o material foi montando entre lâminas e lamínulas seguindo os procedimentos descritos por Christiansen \& Bellinger $(1980,1998)$. Os espécimes foram identificados sob microscópio óptico com contraste de fases.

\section{RESULTADOS}

Foram registradas 54 espécies de colêmbolos, distribuídas em 25 gêneros e 13 famílias, por localidades conforme mostra a Tabela I.

As famílias com os maiores números de gêneros registrados foram Entomobryidae e Paronellidae, com quatro gêneros cada (Tabela I). O maior número de espécies encontradas foi em Entomobryidae, com 22 espécies (40,8\% do total de espécies), seguido por Paronellidae e Dicyrtomidae, com cinco espécies cada $(9,2 \%$ do total cada) (Fig. 2). Apenas três famílias apresentaram uma única espécie: Cyphoderidae, Neelidae e Sminthuridae (Tabela I, Fig. 2).

O gênero mais representativo foi Seira, com 15 espécies registradas $(27,8 \%$ do total de espécies); seguido por Dicyrtoma, com cinco espécies (9,2\% do total); e Lepidocyrtus, Entomobrya e Brachystomella, cada um com três espécies (5,6\% do total cada) (Fig. 3).

A maioria das espécies foi registrada em apenas uma localidade (Tabela I). Apenas Lepidocyrtus nigrosetosus, Seira mirianae, Seira sp. n. 5, Brachystomella agrosa e Cryptopygus thermophilus foram coletadas em mais de uma localidade. Dessas, a espécie de distribuição mais ampla foi $L$. nigrosetosus, encontrada em todas as localidades estudadas (Tabela I).

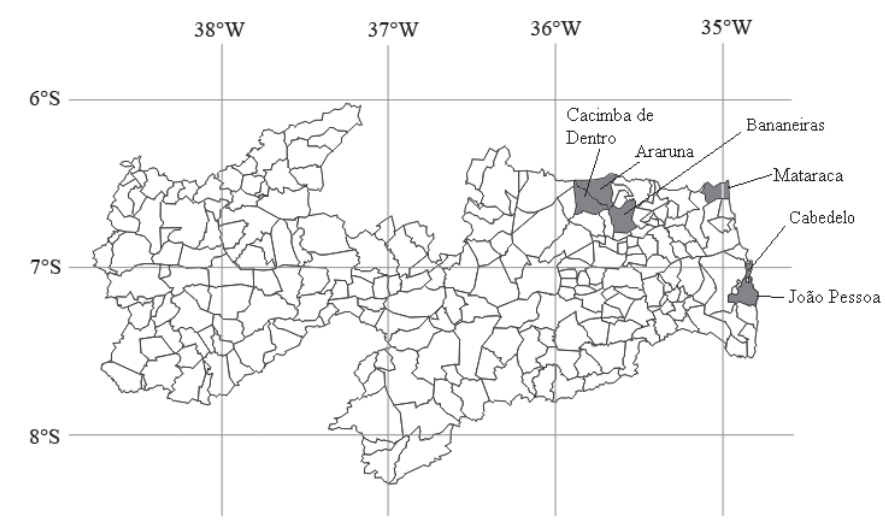

Fig. 1. Mapa do Estado da Paraíba com coordenadas geográficas. Os espécimes foram coletados nos municípios indicados.

A maior parte das espécies de colêmbolos foi encontrada em João Pessoa, Cabedelo e Mataraca, em áreas de remanescentes de Mata Atlântica e Mata de Restinga. Para essas áreas foram contabilizadas 43 espécies (79,6\% do total) (Tabela I). Os municípios de Bananeiras, Cacimba de Dentro e Araruna,onde foram encontradas 14 espécies (25,9\% do total), estão localizados em uma área de transição entre a Mata Atlântica e Caatinga, conhecida por Brejo de altitude (Tabela I).

Os gêneros Pseudosinella, Campylothorax, Arlesia, Sphyroteca, Rastriopes, Sminthurinus e Megalothorax foram registrados pela primeira vez no Estado da Paraíba. As ocorrências de Seira brasiliana, S. prodiga, S. xinguensis, Entomobrya nivalis, E. griseoolivata, Arlesia albipes, Brachystomella parvula, Sphyrotheca mucroserrata e Megalothorax minimus são os primeiros registros dessas espécies para a Paraíba (Culik \& Zeppelini 2003; Zeppelini \&

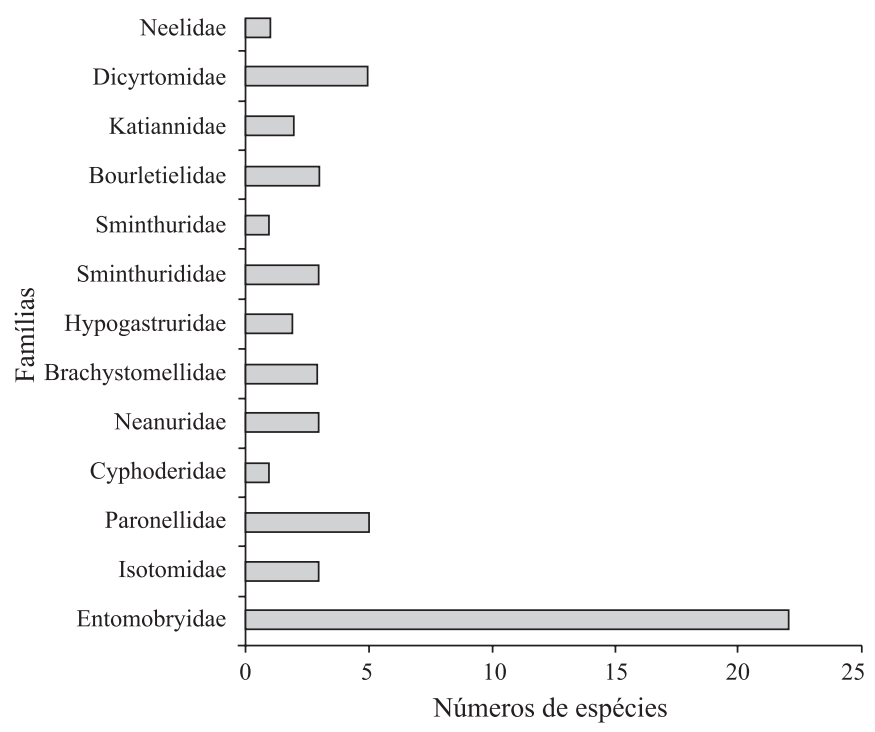

Fig. 2. Número de espécies registradas no Estado da Paraíba distribuído pelas respectivas famílias. 
Tabela I. Espécies de Collembola registradas no Estado da Paraíba, Brasil.

\begin{tabular}{llll}
\hline Táxons $^{1}$ & \multicolumn{2}{c}{ Localidades } \\
\cline { 2 - 4 } & Araruna & Mataraca & João Pessoa Cabedelo Cacimba de Dentro Bananeiras \\
\hline
\end{tabular}

Família Entomobryidae

Entomobrya griseoolivata (Packard, 1873)

Entomobrya nivalis (Linnaeus, 1758)

Entomobrya sp. 1

$\mathrm{x}$

Lepidocyrtus nigrosetosus (Folsom, 1927)

Lepidocyrtus sp.n. 1

Lepidocyrtus sp.n. 2

Pseudosinella sp. 1

Seira arenicola Bellini \& Zeppelini, 2008

Seira brasiliana (Arlé, 1939)

Seira mataraquensis Bellini \& Zeppelini, 2008

Seira mirianae Arlé \& Guimarães, 1981

Seira nigrans (Arlé, 1960)

Seira prodiga (Arlé, 1960)

Seira pseudoannulata Bellini \& Zeppelini, 2008

Seira raptora Zeppelini \& Bellini, 2006

Seira xinguensis (Arlé, 1960)

Seira sp.n. 1

Seira sp.n. 2

Seira sp.n. 3

Seira sp.n. 4

Seira sp.n. 5

Seira sp.n. 6

$\mathrm{X}$

$\mathrm{X}$

$\mathrm{X}$

$\mathrm{X}$

$\mathrm{X}$

$\mathrm{X}$

$\mathrm{X}$

$\mathrm{X}$

$\mathrm{X}$

$\mathrm{X}$

$\mathrm{X}$

$\mathrm{X}$

$\mathrm{x}$

$\mathrm{x}$

$\mathrm{X}$

$\mathrm{X}$

$\mathrm{X}$

$\mathrm{X}$

Cryptopygus thermophilus (Axelson, 1900)

Isotomiella $\mathrm{sp} .1$

Folsomia sp. 1

Família Paronellidae

Lepidonella sp. 1

Lepidonella sp. 2

Paronella sp. 1

Trogolaphysa sp.n. 1

Campylothorax sp. 1

Família Cyphoderidae

Cyphoderus sp.n. 1

$\mathrm{x}$

$\mathrm{x}$

Família Neanuridae

Arlesia albipes (Folsom, 1927)

Neotropiella sp. 1

$\mathrm{x}$

Aethiopella sp. 1

$\mathrm{X}$

$\mathrm{X}$

$\mathrm{x}$

$\mathrm{X}$

$\mathrm{X}$

$\mathrm{X}$

Brachystomella parvula (Schaffer, 1896)

Brachystomella agrosa Wray, 1953

Brachystomella sp. 1

$\mathrm{X}$

Família Hypogastruridae

Xenylla sp. 1

$\mathrm{X}$

Xenylla sp. 2

$\mathrm{X}$

Família Sminthurididae

Sphaeridia heloisae Arlé, 1984

Sphaeridia sp. 1

Denisiella sp.n. 1

$\mathrm{X}$

$\mathrm{X}$ 
Tabela I. Continuação.

\begin{tabular}{lll}
\hline Táxons $^{1}$ & \multicolumn{2}{c}{ Localidades } \\
\cline { 2 - 3 } & Araruna & Mataraca João Pessoa Cabedelo Cacimba de Dentro Bananeiras
\end{tabular}

Família Sminthuridae

Sphyrotheca mucroserrata Snider, 1978

$\mathrm{X}$

Família Borletielidae

Prorastriopes sp.n. 1

Prorastriopes sp.n. 2

Rastriopes sp.n. 1

$\mathrm{X}$

$\mathrm{X}$

$\mathrm{X}$

$\mathrm{X}$

Sminthurinus sp.n. 1

Sminthurinus sp.n. 2

Família Dicyrtomidae

Dicyrtoma sp. 1

Dicyrtoma sp.n. 1

Dicyrtoma sp.n. 2

Dicyrtoma sp.n. 3

Dicyrtoma sp.n. 4

Família Neelidae

Megalothorax minimus Willem, 1900

${ }^{1}$ sp. n. = espécie não descrita

Bellini 2004, Bellini \& Zeppelini 2005). O registro de Sphyroteca mucroserrata é o primeiro para o Brasil.

\section{DISCUSSÃO}

A família Entomobryidae se destacou como a mais diversa no Estado da Paraíba (Tabela I, Fig. 2). Isso se deve, em especial, ao elevado número de espécies do gênero Seira (Tabela I, Fig. 3). O gênero é predominantemente tropical, com 176 espécies descritas (Bellinger et al. 1996-2008, Christiansen \& Bellinger 2000). Anteriormente, para o Brasil, foram registradas 20 espécies de Seira (Culik \& Zeppelini 2003; Zeppelini \& Bellini 2006). O registro de mais seis espécies não descritas (Tabela I) torna o gênero o mais diverso conhecido no país, sendo Sphaeridia o segundo mais diverso, com 20 espécies registradas (Culik \& Zeppelini 2003; Zeppelini \& Bellini 2004).

O elevado número de espécies de Seira encontradas sugere que o Estado da Paraíba seja um hotspot de diversidade para o gênero. Comparativamente, em todo o território dos Estados Unidos e Canadá, apenas seis espécies de Seira foram registradas (Christiansen \& Bellinger 1980, 1998). Embora Seira tenha sido o gênero mais diverso, a maioria das espécies do grupo foi observada em apenas uma localidade, com exceção de Seira sp. n. 5 e $S$. mirianae. Esse padrão foi observado para a maioria das espécies coletadas (Tabela I). Apenas Lepidocyrtus nigrosetosus mostrou ampla distribuição, ocorrendo em todos os municípios amostrados. Esses dados, entretanto, não restringem a distribuição das demais espécies de colêmbolos a áreas específicas. Maiores esforços de amostragem precisam ser feitos para indicar a distribuição das espécies de maneira mais acurada. Mais de $90 \%$ do território do Estado da Paraíba ainda não foi amostrado (Fig. 1).

A maior parte das espécies coletadas (79,6\% do total) foi encontrada em remanescentes de Mata Atlântica e Mata de Restinga. Esse dado reforça a importância da conservação desses biomas, como reserva da biodiversidade de Collembola.

Foram registradas 20 espécies novas de colêmbolos no Estado da Paraíba, equivalendo a $37 \%$ do total de espécies (Tabela I). Isso demonstra a carência de especialistas atuantes na área no Brasil, especialmente no ramo da taxonomia. De

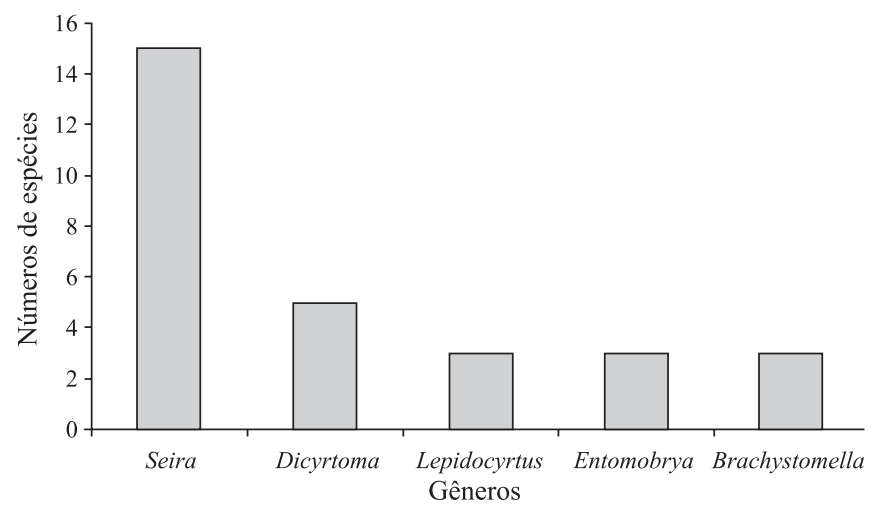

Fig. 3. Número de espécies nos cinco gêneros mais representativos registrados no Estado da Paraíba. 
fato, a real composição da fauna de Collembola no país, especialmente nas regiões Norte e Nordeste, é pobremente conhecida.

Agradecimentos. Agradecemos a Alexandra P. Pais, Claudeci S. Silva, Rodrigo A. Costa, Valderêz H. Costa, Rembrandt R. A. D. Rothéa e Lúcia Raquel R. Berger pela ajuda em campo. O autor Sênior foi bolsista do CNPq.

\section{REFERÊNCIAS}

Abrantes, E. A \& M. C. Mendonça. 2005. Uma nova espécie de Arlea Womersley do sudeste do Brasil (Collembola, Isotomidae). Revista Brasileira de Zoologia 22: 936-939.

Abrantes, E. A \& M. C. Mendonça. 2007. New species and a new Record of Isotomidae (Collembola) from the coast of Brazil. Zootaxa 1500: 55-60.

Bellinger, P. F.; K. A. Christiansen \& F. Janssens. 1996-2008. Checklist of the Collembola of the World. Disponível em: http:// www.collembola.org. [Acessado em 22/08/2008].

Bellini, B. C. \& D. Zeppelini. 2005. First records of Collembola (Ellipura) from the State of Paraíba, Northeastern Brazil. Revista Brasileira de Entomologia 48: $587-588$.

Cassagne, N.; C. Gers \& T. Gauquelin. 2003. Relationships between Collembola, soil chemistry and humus types in forest stands. Biology and Fertility of Soils 37: 355-361.

Cassagne, N.; M. C. Bal-Serin; C. Gers \& T. Gauquelin. 2004. Changes in humus properties and collembolan communities following the replanting of beech forests with spruce. Pedobiologia 48: 267276.

Castaño-Meneses, G.; J. G. Palacios-Vargas \& L. Q. Cutz-Pool. 2004. Feeding habits of Collembola and their ecological niche. Anales del Instituto de Biologia, Universidad Nacional Autónoma de Mexico, Serie Zoologia 75: 135-142.

Choi, W. I. \& D. L. Moorhead. 2006. A modeling study of soil temperature and moisture effects on population dynamics of Paronychiurus kimi (Collembola: Onychiuridae). Biology and Fertility of Soils 43: 69-75.

Christiansen, K. \& P. Bellinger. 1980. The Collembola of North America. North of Rio Grande. Grinnell College, Grinnell, Iowa. $1322 \mathrm{p}$.

Christiansen, K. \& P. Bellinger. 1998. The Collembola of North America. North of Rio Grande. Grinnell College, Grinnell, Iowa. $1322 \mathrm{p}$.
Christiansen, K. \& P. Bellinger. 2000. A survey of the genus Seira (Collembola: Entomobryidae) in the Americas. Caribbean Journal of Science 36: 39-75.

Culik, M. \& D. F. Zeppelini. 2003. Diversity and distribution of Collembola (Arthropoda: Hexapoda) of Brazil. Biodiversity and Conservation 12: 1119-1143.

Fernandes, L. H. \& M. C. Mendonça. 2004. Collembola Poduromorpha do litoral de Marica, Rio de Janeiro, Brasil. Revista Brasileira de Zoologia 21: 15-25.

Frampton, G. K. 2000. Recovery responses of soil surface Collembola after spatial and temporal changes in long-term regimes of pesticide use. Pedobiologia 44: 489-501.

Kooistra, G. 1964. Some data concerning the presence and behavior of springtails (Collembola) on Grass and white clover. Netherlands Journal of Agricultural Science 70: 136-141.

Kristensen, N. P. 1981. Phylogeny of insect orders. Annual Review of Entomology 26: 135-157.

Mari-Mutt, J. A. \& P. F. Bellinger. 1990. A catalog of the Neotropical Collembola. Flora \& Fauna Handbook No. 5. Gainsville, Florida, Sandhill Crane Press. 237 p.

Mari-Mutt, J. A. \& P. F. Bellinger. 1996. Supplement to the Catalog of the Neotropical Collembola - August 1989 to April 1996. Caribbean Journal of Science 32: 166-175.

Mari Mutt, J.A.; P. F. Bellinger \& F. Janssens. 1998-2008. Supplement to the Catalog of the Neotropical Collembola. Disponível em: http://www.collembola.org/publicat/neotrcat.htm. [Acessado em 22/ 08/2008].

Mendonça, M. C. \& L. H. Fernandes. 2007. Rhynchocyrtus gen. nov. (Collembola, Entomobryidae) from the Southeast and Northeast Brazilian regions. Zootaxa 1660: 45-51.

Miranda-Rangel, A. \& J. G. Palacios-Vargas. 1992. Estúdio Comparativo de las comunidades de colémbolos edáficos de bosque de Abies religiosa y cultivo de haba (Vicia faba). Agrociencia, Serie Proteccion Vegetal, 3: 1-14.

Zeppelini, D. F. 2006. The genus Arrhopalites Börner, 1906 (Collembola, Appendiciphora, Arrhopalitidae) in the Neotropical Region, with description of four new cave species from Brazil. Zootaxa 1124: 1-40.

Zeppelini, D. F. \& B. C. Bellini. 2004. Introdução ao estudo dos Collembola. João Pessoa, Paraíba: Editora Universitária, Universidade Federal da Paraíba. 82 p.

Zeppelini, D. F. \& B. C. Bellini. 2006. Two Seira Lubbock 1869 (Collembola, Arthropleona, Entomobryidae) new to science, with remarkable secondary sexual characters. Zootaxa 1185: 21-35.

Recebido em 20/05/2008; aceito em 13/01/2009 